\title{
PHOTOMETRIC AND SPECTROSCOPIC STUDY OF R CMa*
}

\author{
K. R. RADHAKRISHNAN, M. B. K. SARMA, and K. D. ABHYANKAR \\ Centre of Advances Study in Astronomy, Osmania University, Hyderabad, India
}

(Received 19 July, 1983)

\begin{abstract}
U B V$ light curves and spectrograms of R CMa obtained with the 48-inch telescope of JapalRangapur Observatory during 1980-82 have been used for deriving the eclipse and orbital elements as well as the absolute dimensions of the components. The primary is found to be a Main-Sequence F2V star of mass $1.52 M_{\odot}$ and the secondary a subgiant star of spectral type G8 and mass $0.20 M_{\odot}$ which fills its Roche lobe, in agreement with Kopal and Shapley (1956) results, Kopal (1959), or Sahade's (1963) results. From a consideration of the possible evolution of this system it is concluded that a large fraction of the original mass of the secondary is lost from the system. A study of the period changes indicates the possible presence of a third component of mass of about $0.5 M_{\odot}$ which is most likely to be an $M$ dwarf.
\end{abstract}

\section{Introduction}

$\mathrm{R}$ Canis Majoris is the prototype of a group of Algol systems having very low mass function. It is known to exhibit peculiarities in its light curve that change from time to time and is thought to be surrounded by gas. It also shows changes in period which are not yet fully understood. Hence, this star was included in the photometric and spectroscopic observing programme of Japal-Rangapur Observatory.

R CMa was observed photometrically with the 48 -inch telescope in the standard $U B V$ colors on 34 nights during 1980-82 covering nine minima. The individual observations and times of minima have already been published by Radhakrishnan and Sarma (1982). Further 55 spectra of the star with a dispersion of $33 \mathrm{~A} \mathrm{~mm}^{-1}$ at $\mathbf{H} \gamma$ were obtained with the Meinel spectrograph attached to the 48-inch telescope during 1980-81. The results from this data are summarised in this short paper, the details will be published elsewhere.

\section{Analysis of Period Changes}

Dugan (1924), found an abrupt shortening in the period of R CMa in 1914. This was corroborated by Dugan and Wright (1939), Wood (1946), and Guinan (1977). Koch (1960) felt, however, that the (O-C) curve could also be represented by a sine curve of semi-amplitude 0.032 day. We have combined our 9 times of minima

\footnotetext{
* Paper presented at the Lembang-Bamberg IAU Colloquium No. 80 on 'Double Stars: Physical Properties and Generic Relations', held at Bandung, Indonesia, 3-7 June, 1983.
} 
with the 120 other published visual and photoelectric times of minima for making a fresh analysis of the period changes in $\mathrm{R} \mathrm{CMa.}$

On plotting the $(\mathrm{O}-\mathrm{C})$ curve with the second ephemeris of Guinan (1977):

$$
\text { Primary minimum }=\text { HJD } 2420213.1347+1.13593872 \mathrm{E},
$$

an almost sinusoidal variation was noted. After an upward revision of Guinan's period the following revised ephemeris was obtained:

$$
\text { Primary minimum }=\text { HJD } 2430436.5832+1.13594197 \mathrm{E} .
$$

The $(\mathrm{O}-\mathrm{C})$ diagram based on this ephemeris is shown in Figure 1. Its sinusoidal nature indicates apparent change of period either due to apsidal motion or due to the presence of a third body. Since the eccentricity of the binary orbit is almost zero and the secondary minima have always been observed at phase 0.5 the period variation is most likely to be due to the presence of a third body. On applying Irwin's (1952) method and correcting the semi-amplitude by the method of leastsquares we obtained the following elements:

$$
P=29000 \text { binary cycles }=91.44 \mathrm{yr}, e=0.45, \omega=25^{\circ}, K=0^{\mathrm{d}} 029 .
$$

The theoretical $(\mathrm{O}-\mathrm{C})$ curve based on these elements is shown by the solid line in Figure 1. From these elements we obtain

$$
a_{12} \sin i=8.22 \times 10^{8} \mathrm{~km}, \quad f(m)=0.02 M_{\odot}
$$

If we assume a total mass of $1.72 M_{\odot}$ for the binary system the mass of the third

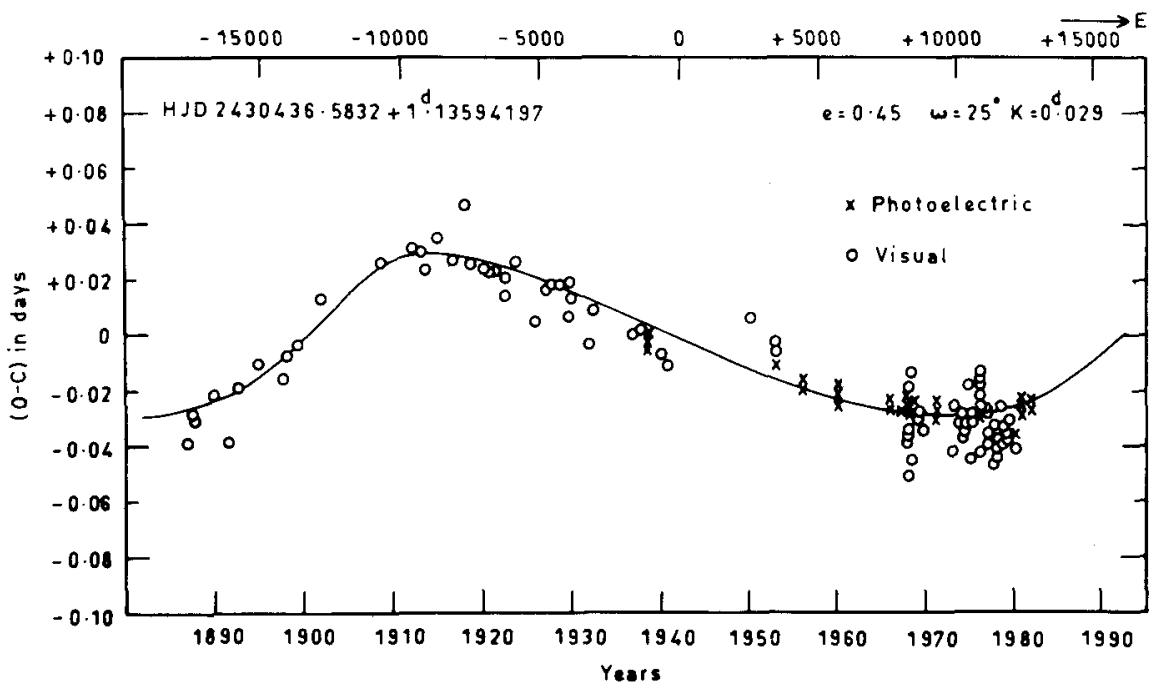

Fig. 1. (O-C) diagram for $\mathrm{R}$ CMa. 
body comes out to be $0.46 M_{\odot}$ for $i=90^{\circ}$ and $0.54 M_{\odot}$ for $i=60^{\circ}$. This low mass suggests that it may be a white dwarf or an $M$ dwarf. Recent findings by Needham et al. (1980) of infrared excess in R CMa may be an indication of the presence of a cool $M$-type third body.

\section{Spectroscopic Orbit}

Fifteen stellar lines were measured for deriving the radial velocities of R CMa. The same lines were also measured on 13 spectra of the radial velocity standard $\alpha$ Lep of spectral type F0. The mean heliocentric velocity of $\alpha$ Lep was found to be $24.2 \pm 0.8 \mathrm{~km}^{-1} \mathrm{~s}$ which is in good agreement with the catalogue value of $24.7 \pm 0.2$ $\mathrm{km} \mathrm{s}^{-1}$.

Preliminary elements of the spectroscopic orbit were calculated both by the method of Russell and Wilsing (Russell, 1902; Wolfe et al., 1967) and by Sterne's (1941) method for a nearly circular orbit. They were corrected by the least squares differential correction method for small eccentricities. Our spectroscopic elements were similar to those derived by the previous authors: Jordan (1916), Sitterly (1940), and Struve and Smith (1950). Hence, all observations were combined to get a common solution by adopting the following procedure:

(i) Phases were calculated by applying the light-time correction due to the motion of the third body.

(ii) The observed radial velocities for each set were corrected for third body motion appropriate for that epoch.

(iii) Differences in the individual systemic velocities for different sets were found to be larger than the corrections for the third body motion; attributing them to the differences in the radial velocity systems all radial velocities were converted to our system.

The final elements are given in Table I and all observations are compared with the theoretical radial velocity curve in Figure 2. The characteristic low mass functions $f(m)=0.0025 M_{\odot}$ is confirmed. The scatter of about $5 \mathrm{~km} \mathrm{~s}^{-1}$ in the observed radial velocities seems to be intrinsic and it may be due to the rotationally broadened and weak lines in the spectrum which limit the accuracy of measurement.

TABLE I

Spectroscopic elements of R CMa

\begin{tabular}{ll}
\hline$e$ & $=0.049 \pm 0.019$ \\
$V_{0}$ & $=37.19 \pm 0.78 \mathrm{~km} \mathrm{~s}^{-1}$ \\
$K_{1}$ & $=27.78 \pm 0.48 \mathrm{~km} \mathrm{~s}^{-1}$ \\
$a_{1} \sin i$ & $=4.33 \pm 0.08 \times 10^{5} \mathrm{~km}$ \\
$\omega$ & $=173^{\circ} .9 \pm 21^{\circ} .2$ \\
$f(m)$ & $=0.00251 \pm 0.00014 M_{\odot}$ \\
\hline
\end{tabular}




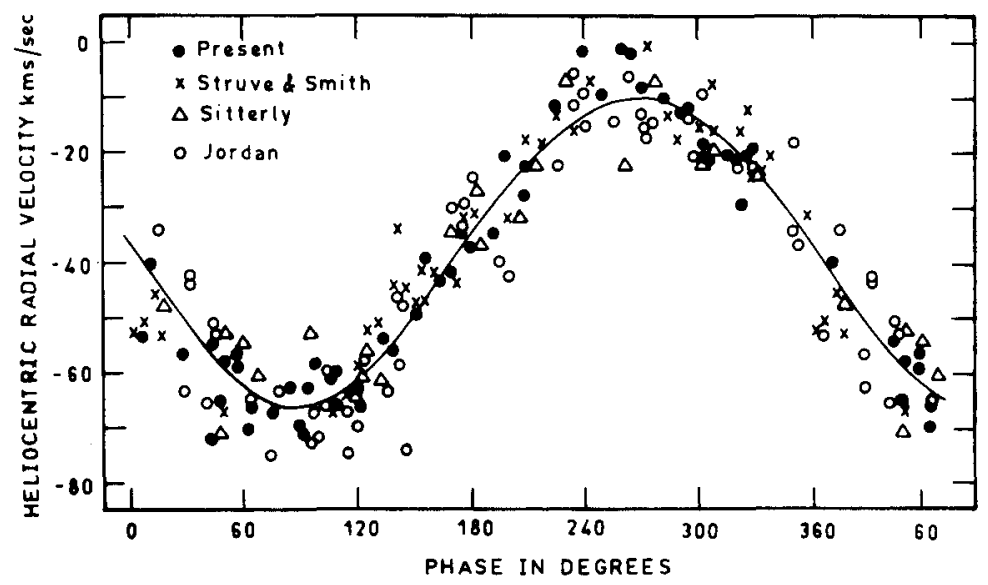

Fig. 2. Radial velocity curve of R CMa.

\section{Photometric Solution and Absolute Dimensions}

Figure 3 shows the $V$ light curve of $\mathrm{R}$ CMa based on 874 observations made on 34 nights; the $B$ and $U$ light curves are similar. Like Wood's (1946) observations our light curves are free from peculiarities of various kinds found by other observers: Pickering (1904), Wendell (1909), Koch (1960), Kitamura and Takahashi (1962), and Sato (1971). Apparently there is no evidence of circumstellar gas at the present epoch.

The $U B V$ light curves were analysed by the standard Russell-Merrill method; the primary eclipse was found to be a transit. The derived inclination and radii of the two components are given in the first row of Table II while the spectral types obtained from the derived colors are given in the fourth row. Now, in order to determine the absolute dimensions of the system we need the mass ratio $m_{2} / m_{1}$ which was estimated in three ways: (i) by assuming that the primary is a MainSequence star of spectral type F2V; (ii) by assuming that the secondary fills its Roche lobe; and (iii) by comparing the observed Fourier coefficients of the light curves with those calculated theoretically from Merrill's (1970) formulae. All of them gave a consistent value of $m_{2} / m_{1}=0.13 \pm 0.01$. The masses and radii obtained by combining it with the spectroscopic elements are given in the fifth and sixth rows of Table II while the absolute bolometric luminosities are given in the last row. Our results confirm the conclusion of Sahade (1963) that R CMa systems are normal semi-detached Algol type binaries. It is found that the secondary, which fills its Roche lobe, is overluminous by about 5 magnitudes and much hotter for its mass. The position of the two components of RCMa in the HR-diagram is shown in Figure 4. 


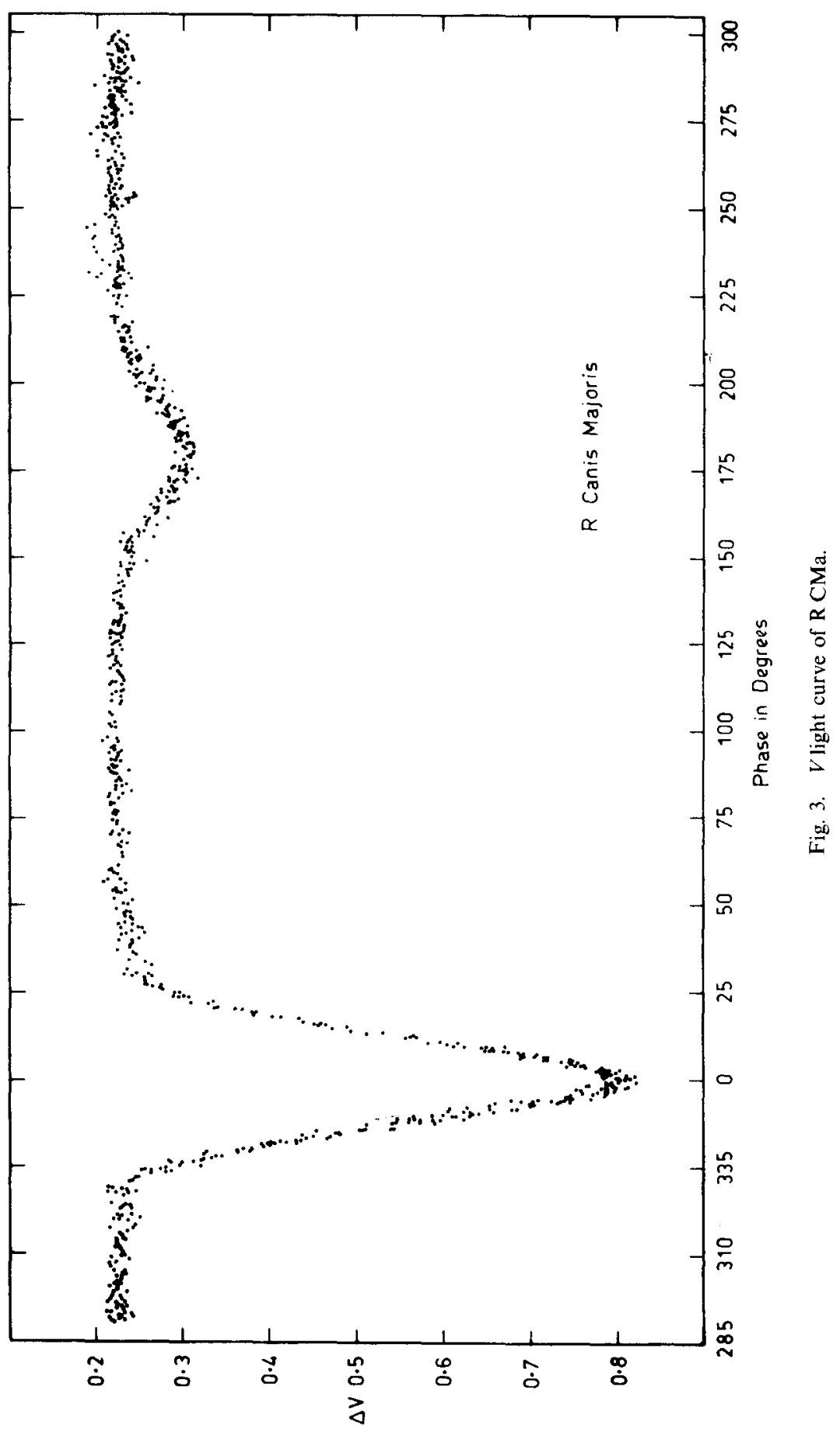


TABLE II

Photometric elements and absolute dimensions of $\mathrm{R} \mathrm{CMa}$

\begin{tabular}{lll}
\hline & Primary & Secondary \\
\hline$i=79^{\circ} .93$ & $r_{h}=0.319$ & $r_{c}=0.217$ \\
$B-V$ & $+0.31+0.01$ & $+0.77 \pm 0.01$ \\
$U-B$ & $+0.04 \pm 0.02$ & $+0.60 \pm 0.02$ \\
Spectral type & F2V & G 8 IV $-\mathrm{V}$ \\
$m_{2} / m_{1}=0.13$ & $m_{h}=1.52 M_{\odot}$ & $m_{c}=0.199 \pm 0.004 M_{\odot}$ \\
$f(m)=0.0025 M_{\odot}$ & $R_{h}=1.73 \pm 0.04 R_{\odot}$ & $R_{c}=1.18 \pm 0.02 R_{\odot}$ \\
$L($ bolometric) & $L_{h}=6.64 \pm 0.29 L_{\odot}$ & $I_{c}=0.86 \pm 0.03 L_{\odot}$ \\
\hline
\end{tabular}

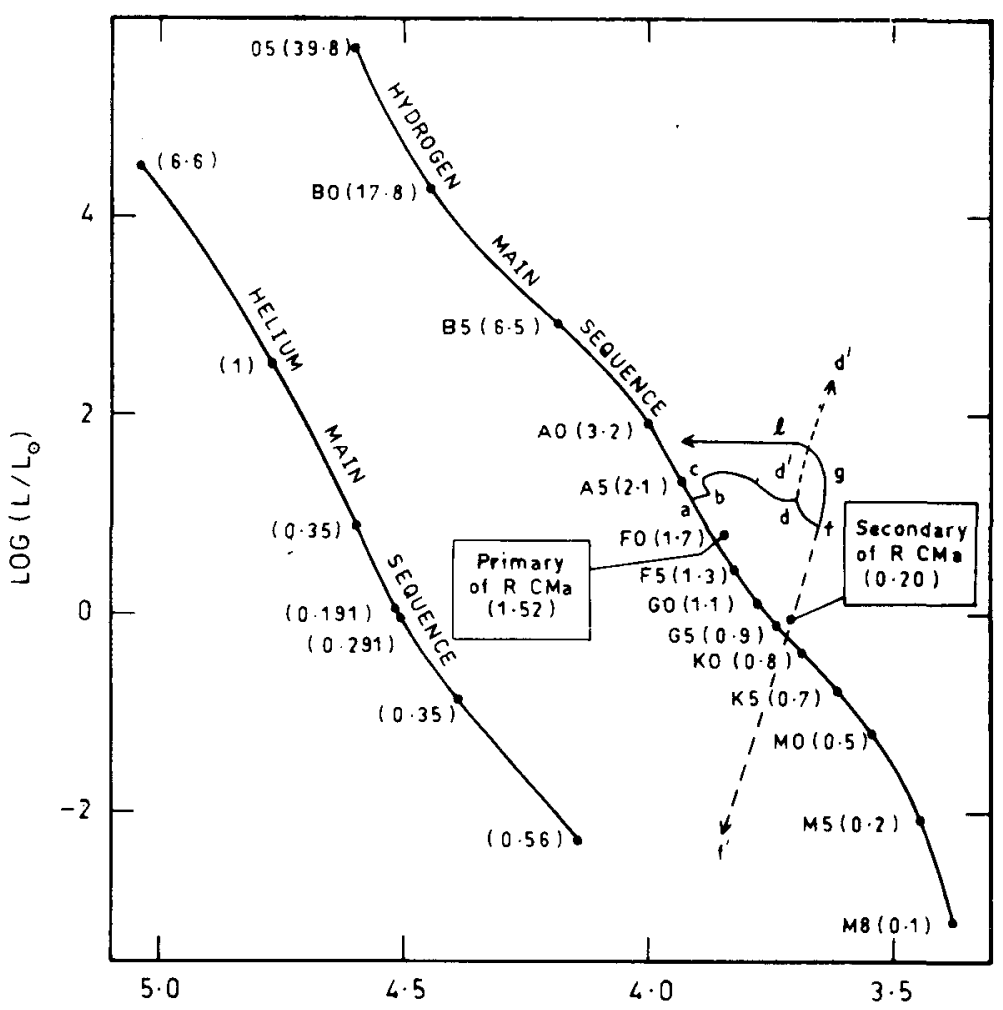

Fig. 4. Position of the components of R CMa in the HR-diagram.

\section{Evolutionary Status of R CMa}

$\mathrm{R} \mathrm{CMa}$ is a typical semi-detached Algol system with a secondary of very low mass. Evolution of such binaries is found to be due to case $B$ mass transfer (Refsdal and 
Weigert 1969; Plavec, 1973). If we assume the mass transfer to be conservative one can start with a system having $M_{1}=1.0$ to $1.2 M_{\odot}$ and $M_{2}=0.7$ to $0.5 M_{\odot}$. Then there are two difficulties: (i) the more massive star would take about $10^{10} \mathrm{yr}$ to reach the stage of case $B$ mass transfer while the system is much younger; according to Guinan and Ianna (1983) it is a high-velocity star of age between 2 to 6 billion years, and (ii) the less massive star cannot absorb a large mass of 0.8 to $1.0 M_{\odot}$ as shows by Neo et al. (1977). An assumption of a complete reversal of mass by taking $M_{1}=1.5 M_{\odot}$ and $M_{2}=0.2 M_{\odot}$ will also not be tenable, because then $M_{2}$ would still be in the gravitational contraction phase and so would not be in a position to receive the matter lost by $M_{1}$. We have, therefore, to conclude that we are dealing with non-conservative mass transfer.

We suggest that the present primary of R CMa would have started with a slightly less mass of $1.3 M_{\odot}$ and the secondary was much more massive with an initial mass of $1.8 M_{\odot}$. The evolutionary track of such a secondary as calculated by Refsdal and Weigert (1969) is shown in Figure 4. The star begins to lose mass at point ' $d$ '. Only a small fraction of the lost mass is absorbed by the companion which has increased its mass to its present value of $1.52 \mathrm{M}_{\odot}$. The remainder is lost from the system through the outer Lagrangian points. However the fact that the star has now apparently moved down from point $f$ instead of going up indicates that it has lost its hydrogen envelope very rapidly and is already on its way to become a helium white dwarf by contraction along the Hayashi track. Similar conclusion about the final stage of evolution of this component is arrived at by Guinan and Ianna (1983).

\section{References}

Dugan, R. S.: 1924, Contr. Princeton Univ. Obs. 6, 49.

Dugan, R. S. and Wright, F. W.: 1939, Contr. Princeton Univ. Obs. 19, 34.

Guinan, E. F.: 1977, Astron. J. 82, 51 ,

Guinan, E. F. and Ianna, P. A.: 1983, Astron. J. 88, 126.

Irwin, J. B.: 1952, Astrophys. J. 116, 211.

Jordan, F. C.: 1916, Alleghany Publ. 3, 49.

Kitamura, M. and Takahashi, C.: 1962, Publ. Astron. Soc. Japan 14, 44.

Koch, R. H.: 1960, Astron. J. 65, 326.

Kopal, Z.: 1959, in Close Binary Systems, Chapman-Hall and John Wiley, London and New York; Chapter VII.

Kopal, Z. and Shapley, M. B.: 1956, Jodrell Bank Annals 1, 143.

Merril, J. E.: 1970, in Vistas Astron. 12, 43.

Needham, J. D., Phillips, J. P., Selby, M. J., and Magro, C. S.: 1980, Astron. Astrophys. 83, 370.

Neo, S., Miyatti, S., Nomoto, K., and Sugimoto, D.: 1977, Publ. Astron. Soc. Japan 29, 249.

Pickering, E. C.: 1904, Harvard Annals 46, 184.

Plavec, M. : 1973, in A. H. Batten (ed.), Extended Atmospheres and Circumstellar Matter in Spectroscopic Binary Systems, D. Reidel Publ. Co., Dordrecht, Holland, p. 216.

Radhakrishnan, K. R. and Sarma, M. B. K.: 1982, Nizamiah and Japal-Rangapur Obs. Contr., No. 16. Refsdal, S. and Weigert, A.: 1969, Astron. Astrophys. 1, 167.

Russell, H. N.: 1902, Astrophys. J. 15, 252.

Sahade, J.: 1963, Ann. Astrophys. 26, 80. 
Sato, K.: 1971, Publ. Astron. Soc. Japan 23, 335.

Sitterly, B. W.: 1940, Astron. J. 48, 190.

Sterne, T. E.: 1941, Proc. U.S. Nat. Acad. Sci. 27, 108.

Struve, O. and Smith, B.: 1950, Astrophys. J. 111, 27.

Wendell O. C.: 1909, Harvard Annals 69, 66.

Wolfe, R. H., Horak, H. G., and Storer, N. W.: 1967, in M. Hack (ed.), Modern Astrophysics, Gauthier-Villars, Paris, p. 251.

Wood, F. B.: 1946, Contr. Princeton Univ. Obs. 21, 31. 